\title{
Terrestrial Magnetism
}

\author{
and \\ Atmospheric Electricity
}

VOLUME VIII

MARCH, I 903

NUMBER I*

\section{DIE BEABSICHTIGTE NORWEGISCHE EXPEDITION}

NACH DEM MAGNETISCHEN NORDPOL. ${ }^{1}$

VON ROALD AMUNDSEN, Leiter der Expedition.

Wenn ich dem für mich so ehrenvollen Ersuchen der Gesellschaft für Erdkunde, über meine beabsichtigte Reise einige Angaben zu machen, gern und mit bestem Danke Folge leiste, so geschieht dies in dem Bewusstsein, dass es für mich nur von dem grössten Nutzen sein kann, vor so hervorragenden Fachleuten meine Pläne zu entwickeln. Ich möchte deshalb die Herren bitten, mir jedes Bedenken gegen das Eine oder das Andere in meinem Vorhaben rückhaltslos zu äussern.

In schwierigen Verhältnissen kann oft ein guter Rath von weittragender Bedeutung sein. Meine Theilnahme an der antarktischen Expedition der Belgica gab mir die erste Veranlassung zu dem Projekt.

Es war ja auf jener Expedition beabsichtigt, zum magnetischen Südpol zu gelangen und die Ausrüstung an magnetischen Instrumenten war dementsprechend eine recht gute. Da jedoch die Expedition zu spät von Süd-Amerika aufbrach, war es nicht mehr möglich über Australien bis in die Gegend des magnetischen Südpols vorzudringen. Wir fuhren von Süd-Amerika direkt nach 1902.

${ }^{1}$ Einen Vortrag gehalten in der Gesellschaft für Erdkunde, Berlin, December 
|I'LTT: 1. |

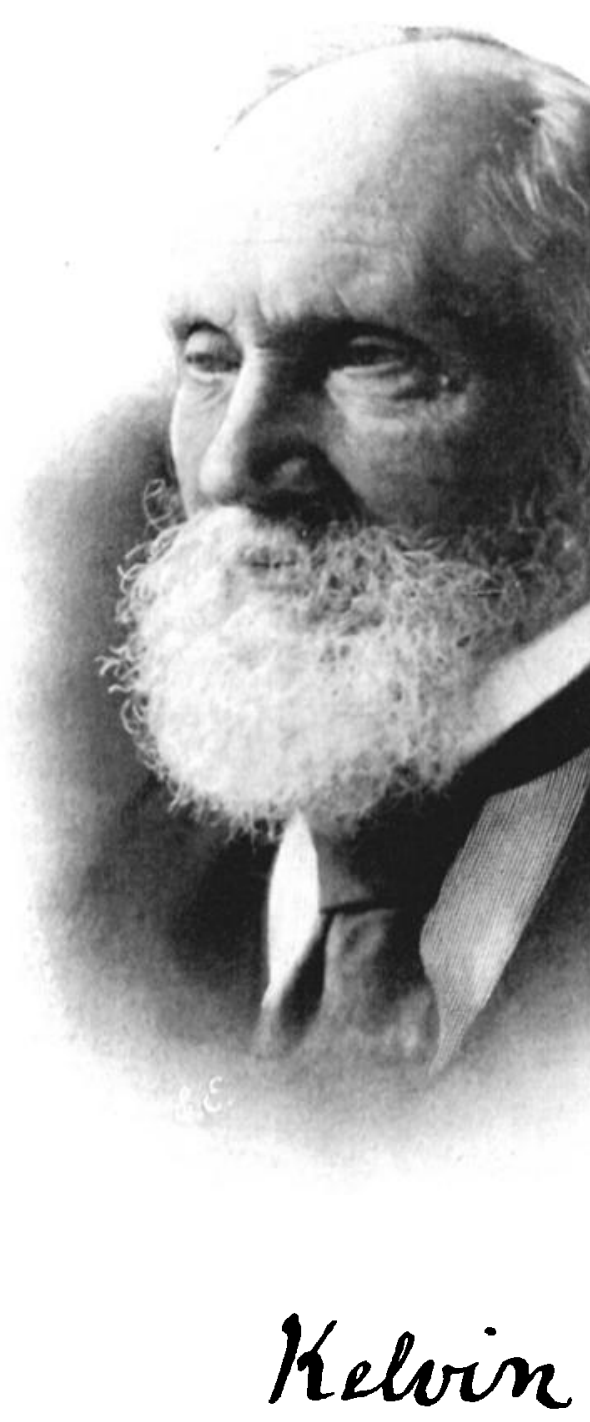


Süden, sassen dann bald in Eise fest und hatten in dieser Situation Zeit und Musse genug, um iber andere Unternehmungen nachzudenken. $\mathrm{Da}$ ich mich an den magnetischen Messungen auf der Belgica betheiligte, wollte ich die gewonnenen Erfahrungen zu einer Erforschung des magnetischen Nordpols weiter verwerthen. Ich habe mich aber bei meinem Vorhaben nur von dem einen Gedanken leiten lassen, womöglich der Wissenschaft einigen Nutzen zu leisten, und aus diesem Grunde einen solchen Reiseweg in Aussicht grenommen, dass ein Erfolg möglichst gesichert wird.

Es liegt mir deshalb fern, unter grossen Schwierigkeiten und Gefahren neue Länder zu entdecken oder vielleicht gar zum astronomischen Norlpol zu gelangen. Wenn ich iiberhaupt den Wunsch hätte, diesen Pol zu finclen, so hätte ich ihn sicher nur cleshalb, um damit allen-ich möchte sagen, sportmässigen-Iixpeditionen dorthin ein Ende zu machen.

Nach der Rückkehr der Belgica wandte ich mich in September 1900 an den Direktor der deutschen Seewarte, Herrn Gelieimrath von Neumayer, und legte ihm meinen Plan vor, in der Gegend des magnetischen Nordpols magnetische, meteorologische und geologische Beobachtungen auszufilliren. Herr Geheimrath stimmte meinem Plan vollständig zu und gab mir während eines Aufenthalts von zwei Monaten an der Seewarte in seiner allbekaunten Liebenswürdigkeit die beste Unterweisung, welche ich haben konnte. Ich finde nicht die Worte, um meine warmen Gefïhle grösster Dankbarkeit für Herrn Geheimrath von Neumayer zum rechten Ausdruck zu bringen. Auf seinen Rath bestellte ich ein magnetisches Reiseinstrument nach seiner Construktion beim Mechaniker Saemann in Hamburg und ein Inclinatorium bei Dover in Kent.

In Norwegen und Scliweden äusserten sich die Herren Prof. Nansen und Prof. Geelmuyden ebenfalls sehr günstig über mein Vorhaben.

Im Jahre Igor kaufte ich dann ein kleines Schiff-Gjöa genannt-für arktische Schifffahrt speciell gebaut.

Um die Eigenschaften des Schiffes gründlich kennen zu lernen, und eventuell noch geeignete Abänderungen zu treffen, unternahm ich mit der Gjöa eine sechsmonatliche Reise nach Novaja Zemblja, Spitzbergen und Grönland. Gleichzeitig machte ich oceanographische Untersuchungen in dem Grönländischen Polarstrom als Abschluss der gleichartigen Beobachtungen von Herrn Prof. Nansen auf dem Fram I903-igo6. 
Im Juni und Juli 1902 war ich in Wilhelmshaven, um die Constanten meiner Instrumente $z u$ bestimmen.

Den Herren Geheimrath Börgen und Prof. Stück bin ich ebenfalls für ihre grosse Unterstützung, welche sie mir andauernd zu Theil werden liessen, zu höchstem Danke verpflichtet.

Zur weiteren Einübung führte ich dann mit meinen Instrumenten in Gemeinschaft mit Herrn Axel Steen an verschiedenen Orten in Norwegen magnetische Messungen aus. An Herrn Prof. Schmidt hatte ich mich bereits früher um Rath gewandt, damit ich in kompetentester Weise darüber unterrichtet war, welche Messungen ich auszufïhren hatte, um ihnen vom theoretischen Standpunkt aus den grössten Werth zu verleihen. Da ich beabsichtige, auch magnetische Registrirungen mit Instrumenten nach Eschenhagen durchzufuihren, suclite ich Herrn Prof. Schmidt am magnetischen Observatorium zu Potsdam auf, um die Instrumente zu bestellen und gleichzeitig die Constanten der magnetischen Instrumente, welche bei der letzten Fram Expedition benutzt wurden und welche ich ebenfalls zu verwerthen gedenke, neu zu prüfen.

Auf den Rath des Herrn Prof. Schmidt gedenke ich die BasisStation-von der aus dann kleinere Expeditionen unternommen werden sollen-den magnetischen Pol nicht allzu nahe zu errichten, damit daselbst alle magnetischen Elemente noch mit grösserer Sicherheit bestimmt werden können und damit besonders die Veränderlichkeit der Declination, genaue Messungen nicht gar zu sehr erschwert.

Ich werde daselbst die Declination, Horizontal-Intensität und Vertical-Intensität ganz in der Weise registriren, wie sie von Herrn Prof. Eschenhagen im "Terrestrial Magnetism" beschrieben ist. Die drei Instrumente stehen hintereinander, erhalten von einer Lampe Licht und registriren dann ihren Stand zugleich auf dem photographischen Papier einer Walze. Nur wird es sich für meine Zwecke empfehlen, die Entfernungen der Instrumente etwas anders zu wählen.

Die absoluten magnetischen Messungen werden während der Winterzeit mit zwei Reiseinstrumenten, einem Fox Apparat und einem Erdinductor nach Wild-Eschenhagen ausgeführt werden. In den kleineren Expeditionen werde ich nur das eine Reiseinstrument und vielleicht auch den Fox Apparat mitnehmen.

Wie ich dies bereits vor einem Jahre in einem Vortrage in Christiania hervorgehoberi habe, werde ich mich im wesentlichen 
darauf beschränken müssen, den magnetischen Pol in einem beträchtlichen Abstand von mindestens $200 \mathrm{~km}$. zu umkreisen, da die Beobachtungen in nächster Nähe des Pols zu unsicher sein werden und zı grossen Täuschungen Veranlassung geben könnten. Die Messungen wïrden eigentlich nur als Local-Untersuchungen zu bezeichnen sein, welche um so weniger allgemeinen Werth haben, als daselbst infolge der grösseren Total-Intensität die LocalStörungen voraussichtlich recht erheblich sein werden und infolge des geringen absoluten Werthes der Horizontal-Intensität viel mehr zur Geltung kommen als anderswo. Genaue Messungen würden dort auch mit mancherlei Schwierigkeiten mehr instrumenteller Natur verknüpft sein; das Mess-Instrument müsste im grossen und ganzen mehr als Local-Variometer ausgestattet sein.

Aus instrumentellen Gründen werde ich zur grösseren Sicherheit eine Anzahl Magnete von verschiedenstem Moment mitnehmen. Dieselben sollen so in eisernen Behältern verpackt sein, dass sie ihre Momente erfahrungsgemäss sehr constant erhalten. Dann bin ich in der Lage, die Horizontal-Intensität entweder durch Ablenkungen oder durch Schwingungen allein zu messen.

Messungen ersterer Art werden besonders während der Schlitten-Expeditionen vorzuziehen sein, während sich der Anschluss meiner Basis-Station an Potsdam hinsichtlich der HorizontalIntensität vielleicht am besten durch Schwingungs-Beobachtungen ausführen lässt. Ich beabsichtige aber etappenweise von hier aus bis zu meiner Station Messungen auszufihren, un die AblenkungsConstanten von Magneten, welche sich hier wegen der kleinen Ablenkungs-Winkel nicht mit genügender Sicherheit bestimmen lassen, unterwegs $z \mathbf{u}$ ermitteln.

Die Gjöa, das Expeditionsschiff, ist, wie gesagt, nur klein, aber gerade dadurch wie auch durch ihre starke Bauart besonders für die Eisregion geeignet; ich hoffe mit ihr leichter als mit einem grösseren Schiffe durch die schmalen Fahrstrassen jener Gewässer 2u kommen, welche ich durchforschen will. Das Schiff ist 70 Fuss lang, 20 Fuss breit und hält 48 Registertons, ich habe es aber noch durch schwere Holz- und Eisenbalken verstärken lassen. Auch ist es jetzt mit einem kleinen Petroleummotor versehen und läuft mit diesem etwa 4 Knoten in der Stunde. 30,000 Liter Petroleum in eisernen Behältern sollen zur Heizung, zum Kochen und als Triebkraft fitr den Motor dienen. Das Petroleum wird aber grossenteils durch schottische Walfishfänger bis in die Nähe der Gegend gebracht, wo ich voraussichtlich zunächst überwintern werde. 
Proviant nehme ich für 4-6 Jahre mit, sowie eine möglichst gute Ausrüstung für eine Polar-Expedition.

Die Besatzung wird aus acht Mann bestehen, von denen zweiein Assistent und ich-im wesentlichen die magnetischen Arbeiten ïbernehmen, während ein dänischer Marine-Officier hauptsächlich geologische Studien machen wird.

Von Christiania werde ich voraussichtlich am I5. April rgo3 aufbrechen. In Godhavn, auf der Insel Disco, auf der Westseite von Grönland, will ich mich mit Polarhunden versehen und magnetische Messungen ausfihien. Solche Messungen sollen weiterhin an allen Orten, wo es möglich ist, angestellt werden, damit ich daraus auf die gegenwärtige Lage des magnetischen Nordpols Schlüsse ziehen kann. Wie mir mehrere Walfischfahrer mittheilten, fangen bereits in der Lancaster Strasse die besten Compasse von William Thomson an, ganz unzuverlässig $z \mathfrak{u}$ werden.

Nach den bisherigen Ueberlegungen werde ich die Basis-Station am Leopold Hafen auf Nord Somerset einrichten. Da jedoch für die Wahl dieser Station mancherlei mitspricht, worïber ich vorläufig selber nichts Näheres weiss, kann ich natürlich keine ganz bestimmten Angaben über die Lage dieser Station machen. Auf jeden Fall möchte ich versuchen, im Sommer 1904 durch schottische Walfischfahrer, welche diesen Hafen gewöhnlich aufsuchen, Nachricht von mir nach Europa gelangen zu lassen. Auf der Station wird im Spätsommer und während des Winters mit der Einrichtung, sowie mit den absoluten Messungen und anderen Beobachtungen genügend zu thun sein. Der Raum für das RegistrirObservatorium wird im wesentlichen aus leeren Proviantkisten hergestellt werden; mit den Wohnräumen soll dieses Observatorium durch einen allseitig gedeckten Gang verbunden sein, um auch bei starken Schneeverwehungen noch sicher und bequem dorthin zu gelangen. Das absolute Observatorium wird im Winter aus einer Schneehïtte bestehen. Im Sommer werde ich dazu ein festes Zelt benutzen, dessen Decke aus durchsichtiger weisser Seide besteht, wodurch ich genügendes Oberlicht für die Messungen erhalte. Während des Sommers gedenke ich dann eine Anzahl von Schlittenexpeditionen auszuführen, um einen möglichst grossen Theil des Gebiets rings um den magnetischen Pol zu vermessen. Ich will in diesem Sommer auch versuchen, mit dem Schiff einen Weg nach King William Insel zu finden, um im zweiten Sommer das Observatorium dorthin $z \mathfrak{u}$ verlegen und nun von dort aus neue kleinere Expeditionen auszufuibren. Im dritten Sommer 
also 1906 will ich dann versuchen, in der Gegend der Herschel Insel meine Basis-Station zu errichten. Dort hoffe ich wohl Gelegenheit zu haben, mit McPherson-dem nördlichsten Fort der britischen Handels-Compagnie-in Verbindung zu treten. Hier sollen dann die Messungen ganz ebenso wie in den Jahren vorher fortgesetzt werden. Nach Ablauf der Zeit, welche ich mir gesetzt habe, werde ich dann die Rückreise um Alaska herum antreten und womöglich in Sitka Anschlussmessungen am magnetischen Observatorium der Vereinigten Staaten "Coast and Geodetic Survey" machen.

Ich schliesse meine Mittheilung, indem ich der Gesellschaft nochmals meinen Dank für die freundliche Einladung ausspreche. 